\title{
Nucleic Acid Analysis Using Multifunctional Hybridization Sensors ${ }^{\dagger}$
}

\author{
Dmitry M. Kolpashchikov ${ }^{1,2}$ \\ 1 Chemistry Department, University of central Florida, Orlando, FL 32816, USA; \\ Dmitry.Kolpashchikov@ucf.edu \\ 2 Laboratory of Solution Chemistry of Advanced Materials and Technologies, ITMO University, \\ Lomonosova St. 9, 191002 Petersburg, Russia \\ + Presented at the 5th International Symposium on Sensor Science (I3S 2017), Barcelona, Spain, \\ 27-29 September 2017.
}

Published: 24 November 2017

Hybridization of nucleic acid probes remains one of the most common strategies for sensing of specific DNA and RNA sequences. Formats that use hybridization probes include qualitative PCR, microarrays, fluorescent in situ hybridization (FISH), to name a few. Moreover, specific recognition of RNA sequences is in demand for gene silencing approaches, e.g., antisense and siRNA. Hybridization probes are nucleic acid oligomers of 15-25 nucleotides (or longer) designed to be complementary to targeted analytes. The formation of a probe-analyte hybrid testifies that the analyte contains a nucleotide sequence complementary to the probe. This approach suffers from low selectivity (especially for temperatures $<40{ }^{\circ} \mathrm{C}$ ), high cost for fluorescent probes, and poor target accessibility if folded natural RNA are analyzed. Part of the sensing problem arises from the affinity/selectivity dilemma: the higher the probe-analyte affinity, the lower the selectivity. To address these and other problems, we design multicomponent hybridization probes (MHP) that consist of several oligonucleotide components, which associate with the RNA/DNA target and produce a detectable signal. Each MHP component serves a specific function, thus enabling simultaneous improvement of multiple key characteristics. My presentation will cover the design of a probe that can differentiate single nucleotide substitutions in DNA in the entire temperature interval of $5-40^{\circ} \mathrm{C}$ [1]; molecular machine that tightly binds the RNA analyte while remaining highly selective [2]; a strategy for the recognition of highly variable viral genomes with high selectivity [3].

Acknowledgments: Funding from NSF CCF 1423219 and NIH 1R15AI103880-01A is greatly appreciated. D.M.K. was partially supported by the ITMO University Fellowship and Professorship Program.

Conflicts of Interest: The author declare no conflict of interest.

\section{References}

1. Stancescu, M.; Fedotova, T.A.; Hooyberghs, J.; Balaeff, A.; Kolpashchikov, D.M. Nonequilibrium Hybridization Enables Discrimination of a Point Mutation within 5-40 ${ }^{\circ}$ C. J. Am. Chem. Soc. 2016, 138, 13465-13468.

2. Cox, A.J.; Bengtson, H.N.; Rohde, K.H.; Kolpashchikov, D.M. DNA nanotechnology for nucleic acid analysis: multifunctional molecular DNA machine for RNA detection. Chem. Commun. 2016, 52, 1431814321.

3. Kamar, O.; Sun, S.C.; Lin, C.H.; Chung, W.Y.; Lee, M.S.; Liao, Y.C.; Kolpashchikov, D.M.; Chuang, M.C. A mutation-resistant deoxyribozyme OR gate for highly selective detection of viral nucleic acids. Chem. Commun. 2017, 53, 10592-10595. 